\title{
Characterization of Atherosclerotic Plaques by NIRS-IVUS System
}

\author{
Leone Simona* \\ Department of Chemistry, Università dell'Aquila, Italy \\ *Corresponding author: Leone Simona, Department of Chemistry, Università dell'Aquila, Italy. \\ To Cite This Article: Leone Simona. Characterization of Atherosclerotic Plaques by NIRS-IVUS System. Am J Biomed Sci \& Res. 2019 - 3(6). \\ AJBSR.MS.ID.000733. DOI: 10.34297/AJBSR.2019.03.000733
}

Received: June 28, 2019 | Published: July 11, 2019

\section{Introduction}

Nowadays, the pathologies of the cardiovascular system represent the main cause of mortality in our country: about $45 \%$ of all deaths are, in fact, attributable to cardiovascular diseases. In particular, of the 250,000 cases of annual death due to cardiovascular diseases, two-thirds are attributable to coronary heart disease. Atherosclerosis is the vascular pathology with the greatest clinical impact because it is responsible for myocardial infarction and cerebrovascular accidents that represent the main cause of death and morbidity in industrialized countries. Coronary atherosclerosis is a multifactorial pathology that, to become clinically manifest, requires the formation of a fibro-lipid plaque inside the wall of an artery that reduces blood flow; it is a dynamic degenerative disease that can have a sudden transition from a chronic condition to clinical instability following a process of plaque rupture and thrombotic overlap. The primary objective of the research remains the early identification of the lesion at risk of instability in order to prevent the clinical event. This lesion on the path of instability is described as a "vulnerable plaque", i.e. a lesion with a high probability of becoming responsible for an acute event in the future.

The desire to identify and treat vulnerable plaques before major adverse events stimulated the development of invasive and non-invasive high-resolution imaging techniques.

Carotid ultrasonography was the first technique used to identify asymptomatic subjects a critical subclinical pathology or very early alterations, such as an abnormal thickening of the intimate and medium coats. Over the years these simpler techniques have been accompanied by other more complex methods such as computerized multi-layer tomography (MSCT), magnetic resonance imaging (MRI) or other invasive evaluations, such as angiography, intracoronary ultrasound (IVUS, Intravascular Ultrasound), optical coherence tomography (OCT), to capture even the finest characteristics of atherosclerotic plaques and try to assess the risk of progression or instability more precisely. A critical analysis of these different diagnostic methods is appropriate as these are techniques useful for identifying patients with high atherosclerotic load or instruments aimed at identifying plaques at greater risk of rupture and therefore cause of acute coronary events [1-4].

To date, no imaging technique can accurately identify the risk of plaque rupture. It is therefore essential to develop alternative imaging techniques capable of obtaining a correct typing of the atherosclerotic plaque to identify the «vulnerable», with a high risk of breakage

In several studies it was analyzed the possibility of using near-infrared spectroscopy (NIRS, Near Infrared Spectroscopy), a routine technique used in physics to determine the chemical composition of substances, to identify the chemical components of coronary plates as a means to evaluate their vulnerability. The goal of intracoronary NIR spectroscopy is to produce a "chemogram" of the artery wall that will serve as a vulnerability index. Studies conducted by different groups have definitively documented that, in the absence of difficulties posed by cardiac movement and the presence of blood, NIR spectroscopy, can accurately identify the important characteristics of atherosclerotic plaques suspected of representing plaque vulnerability [5-7].

In 2009, Waxman et al. [8] conducted the first human studies to test the safety and efficacy of NIRS spectroscopy in the detection of coronary plaques with lipid core.

NIR spectroscopy applied to the coronary arteries is a promising diagnostic imaging modality that plays a key role in interventional cardiology practice, allowing a better and early diagnosis of coronary diseases. The identification of the composition is important because the coronary arteries can contain plaques with lipid core that can lead to heart attacks and complications in stenting procedures.

Stenting is traditionally guided by an X-ray angiography that identifies the general position of the lumen narrowing but cannot reveal the composition of the vessel wall. To overcome the limitations of angiography, doctors turn to various advanced 
imaging solutions to optimize not only their stenting strategy, but also the care their interventional cardiology patients must undergo.
Several studies have compared different intracoronary imaging technologies for the detection of atherosclerotic plaques (Table 1).

\begin{tabular}{|c|c|c|c|c|c|c|}
\hline Table 1: Imaging technologies for the detection of atherosclerotic plaques. & Angiography & Angioscopy & OCT & IVUS & NIRS & NIRS-IVUS \\
\hline Cap Thickness & & 0 & $v$ & 0 & 0 & 0 \\
\hline Expansive Remodeling & & & & $v$ & $v$ \\
\hline Volume plate & & & & $v$ & $v$ \\
\hline Calcification & $v$ & & 0 & $v$ & \\
\hline Trombus & 0 & $v$ & $v$ & 0 & 0 & 0 \\
\hline Macrophage Inflammation & & & 0 & & & \\
\hline Lipidus Core & & 0 & 0 & 0 & $v$ & $v$ \\
\hline Field Visual Free from Blood & NO & SI & SI & NO & NO & NO \\
\hline v: Direct, Sturdy, Validated; O: Indirect, Not Sturdy, Not Valid & & & & & \\
\hline
\end{tabular}

Table 2: Imaging methods for the detection of an introvascular plaque.

\begin{tabular}{|c|c|c|c|c|c|c|}
\hline & Angiography & Angioscopy & OCT & IVUS & NIRS & TVC(NIRS-IVUS) \\
\hline hood thickness & & 0 & $v$ & 0 & 0 & 0 \\
\hline Expansive Remodeling & & & & $v$ & & $v$ \\
\hline Volume plate & & & & $v$ & & $v$ \\
\hline Calcification & $v$ & & 0 & $v$ & & $v$ \\
\hline Trombus & 0 & $v$ & $v$ & 0 & 0 & 0 \\
\hline Macrophage Inflammation & & & 0 & & & \\
\hline Lipidus Core & & 0 & 0 & 0 & $v$ & $v$ \\
\hline visual change free from blood & NO & SI & SI & NO & NO & NO \\
\hline \multicolumn{7}{|c|}{ v: Direct, Sturdy, Validated; O: Indirect, Not Sturdy, Not Valid } \\
\hline
\end{tabular}

The ideal invasive device for the characterization of coronary plaques should provide a complete roadmap of the atherosclerotic load throughout the coronary artery and detect specific lesion data that characterizes the structure, composition and biological dynamics of each plaque (Table 2).

Imaging techniques, such as angiography, IVUS, OCT, and angioscopy, provide valuable insights into structure and function, but are unable to provide accurate and easily obtainable information regarding plaque composition.

Unlike IVUS and OCT, which detect a lipid core based on signal loss, NIRS spectroscopy allows the direct detection of the lipid core since cholesterol has a unique spectral identity in the NIR spectrum that allows its differentiation from other biological components. Since NIRS spectroscopy provides only composition data, it can be used to supplement the structural data provided by angiography, IVUS and OCT.

While the devices previously available were able to achieve many of these objectives in a reproducible, safe and economically advantageous way, different characteristics were not detectable, such as the ability to accurately identify an LRP (Lipid-Rich Plaque) and to quantify the degree of inflammation. These additional characteristics are, instead, available combining the intracoronary IVUS and the NIRS, since a combination of these provides a complete characterization of the structure and composition of the vessel through the system's ability to detect plaques with a high lipid load.

This complete characterization of the vessel promotes optimal clinical decision-making and supports more effective treatment strategies; for example, the NIRS will be able to confirm the presence of LCP if the high plaque load and the hypoechoic regions are detected by the IVUS, which could indicate lesions with a high risk of distal embolization during balloon dilation and stenting [9].

Intravascular ultrasound imaging (IVUS) provides an image of the structure of the wall but has limited ability to determine the composition of the plaques. An important feature of NIRS is that light can penetrate into the tissues and can therefore identify and differentiate different types of tissue, despite the presence of blood between the detector and the target tissue. This is an important advantage for coronary artery imaging.

NIR spectroscopy rapidly, specifically, and reliably identifies the lipid core plates and the aforementioned information combined with those related to the vessel structure obtained by IVUS allow a true vessel characterization to be performed.

This complete characterization of the vessel promotes optimal clinical decision-making and supports more effective treatment strategies; for example, the NIRS will be able to confirm the 
presence of LCP if the high plaque load and the hypoechoic regions are detected by the IVUS, which could indicate lesions with a high risk of distal embolization during balloon dilation and stenting.

For the reduction of coronary risk, early identification and stabilization of atherosclerotic plaques is of primary importance; the imaging of atherosclerosis has therefore an important clinical role today, far superior to its very great scientific utility and the combination of near-infrared spectroscopy and coronary intravascular ultrasound, allowing a better and early diagnosis of coronary diseases, represents one of the most promising technologies in monitoring and identifying the vulnerability of atherosclerotic plaques to future interventional cardiology.

\section{References}

1. Dempsey RJ, Davis DG, Robert G Buice, Robert A Lodder (1996) Biological and medical applications of near-infrared spectroscopy. Appl Spectroscopy 50(2): 18A-34A.

2. Cassis LA, Lodder RA (1993) Near-IR imaging of atheromas in living arterial tissue. Anal Chem 65(9): 1247-1256.

3. Jaross W, Neumeister V, Lattke P, Schuh D (1999) Determination of cholesterol in atherosclerotic plaques using near infrared diffuse reflection spectroscopy. Atherosclerosis 147(2): 327-337.
4. Neumeister V, Scheibe M, Lattke P, Jaross W (2002) Determination of the cholesterol-collagen ratio of arterial atherosclerotic plaques using near infrared spectroscopy as a possible measure of plaque stability. Atherosclerosis 165(2): 251-257.

5. Wang J, Geng YJ, Guo B, Klima T, Lal BN, et al. (2002) Near-infrared spectroscopic characterization of human advanced atherosclerotic plaques. J Am Coll Cardiol 39(8): 1305-1313.

6. Moreno P, Lodder R, Purushothaman K, William E Charash, William N O'Connor, et al. (2002) Detection of lipid pool, thin fibrous cap, and inflammatory cells in human aortic atherosclerotic plaques by nearinfrared spectroscopy. Circulation 105: 923-927.

7. Moreno PR, Ryan SE, David W Hopkins, K Raman Purushothaman, Barry $M$, et al. (2002) Identification of lipid-rich plaques in human coronary artery autopsy specimens by near-infrared spectroscopy. J Am Coll Cardiol 37(Suppl 2): sA356.

8. Waxman S, Dixon SR, L'Allier P, Moses JW, Petersen JL, et al. (2009) In vivo validation of a catheter-based near-infrared spectroscopy system for detection of lipid core coronary plaques: initial results of the SPECTACL study. JACC Cardiovasc Imaging 2(7): 858-868.

9. Stary HC, Chandler AB, Glagov S, Guyton JR, Insull W Jr, et al. (1994) A definition of initial, fatty streak, and intermediate lesions of atherosclerosis: a report from the Committee on Vascular Lesions of the Council on Arteriosclerosis, American Heart Association. Special report, Arterioscler Thromb 14(5): 840-856. 